\title{
Extension of electron cyclotron heating at ASDEX Upgrade with respect to high density operation
}

\author{
Martin Schubert ${ }^{1,}$, Jörg Stober ${ }^{1}$, Albrecht Herrmann ${ }^{1}$, Walter Kasparek $^{2}$, Fritz Leuterer ${ }^{1}$, Francesco Monaco ${ }^{1}$, \\ Bernhard Petzold ${ }^{1}$, Burkhard Plaum $^{2}$, Stefan Vorbrugg ${ }^{1}$, Dietmar Wagner ${ }^{1}$, Hartmut Zohm ${ }^{1}$, \\ and the ASDEX Upgrade Team ${ }^{1}$ \\ ${ }^{1}$ Max Planck Institute for Plasma Physics, Boltzmannstr. 2, 85748 Garching b. München, Germany
${ }^{2}$ Institut für Grenzflächenverfahrenstechnik und Plasmatechnologie, Pfaffenwaldring 31, D-70569 Stuttgart
}

\begin{abstract}
The ASDEX Upgrade electron cyclotron resonance heating operates at $105 \mathrm{GHz}$ and $140 \mathrm{GHz}$ with flexible launching geometry and polarization. In 2016 four Gyrotrons with $10 \mathrm{sec}$ pulse length and output power close to $1 \mathrm{MW}$ per unit were available. The system is presently being extended to eight similar units in total. High heating power and high plasma density operation will be a part of the future ASDEX Upgrade experiment program. For the electron cyclotron resonance heating, an O-2 mode scheme is proposed, which is compatible with the expected high plasma densities. It may, however, suffer from incomplete single-pass absorption. The situation can be improved significantly by installing holographic mirrors on the inner column, which allow for a second pass of the unabsorbed fraction of the millimetre wave beam. Since the beam path in the plasma is subject to refraction, the beam position on the holographic mirror has to be controlled. Thermocouples built into the mirror surface are used for this purpose. As a protective measure, the tiles of the heat shield on the inner column were modified in order to increase the shielding against unabsorbed millimetre wave power.
\end{abstract}

\section{Introduction}

Progress has been made at the ASDEX Upgrade tokamak in controlling high power $\left(\mathrm{P}_{\text {heat }} \approx 20 \mathrm{MW}\right)$ discharges, where the power normalized to the major radius $\left(\mathrm{R}_{0}=1.65 \mathrm{~m}\right)$ is approaching ITER relevant values [1]. A solid tungsten divertor allows to gain experience in operation under ITER-like heat loads [2]. Accumulation of tungsten in the plasma center can be effectively avoided, if the central heating power density is high enough [3,4]. At ASDEX Upgrade (AUG) the electron cyclotron resonance heating (ECRH) can serve for this purpose. This system routinely operates at 105 and $140 \mathrm{GHz}$ [5] and it is used for various wave heating schemes and current drive. Movable launcher mirrors and a broad-band polarization system allow for flexible launching geometries and heating schemes.

With respect to high density and high heating power operation, an O-2 mode scheme at $140 \mathrm{GHz}$ has been developed [6]. This scheme extends the range of accessible plasma densities above the X-2 cut-off, however it may suffer from reduced single pass absorption of the order of $60-75 \%$ [7]. Installing reflectors into the heat shield on the inner column of AUG improves the situation significantly, since the unabsorbed fraction makes a second pass through the plasma. Several constraints on the reflector design had to be taken into account and this finally led to the holographic or phase grating mirror design [8].
This paper will give an overview of the current status of the AUG ECRH installations in the next section. The $\mathrm{O}-2$ mode scheme is prepared by simulations, which are described in section 3. Some points of the holographic mirror design, manufacturing and instrumentation are considered in section 4. Protective measures for the AUG machine are explained in section 5 and with a few comments on ECRH operation with the holographic mirrors this paper shall close.

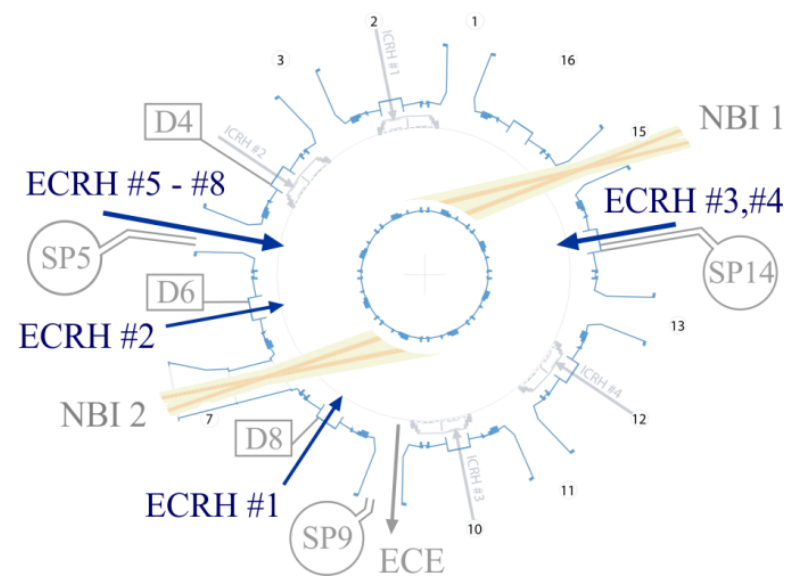

Fig. 1. Top view of equatorial cross section through the AUG vacuum vessel with launch positions of heating systems. ECRH relevant detectors for unabsorbed millimetre waves are also shown in light colour. 'SP' Sniffer sphere, 'D' D-band pick-up waveguide, mounted on quartz window $[12,13]$. 


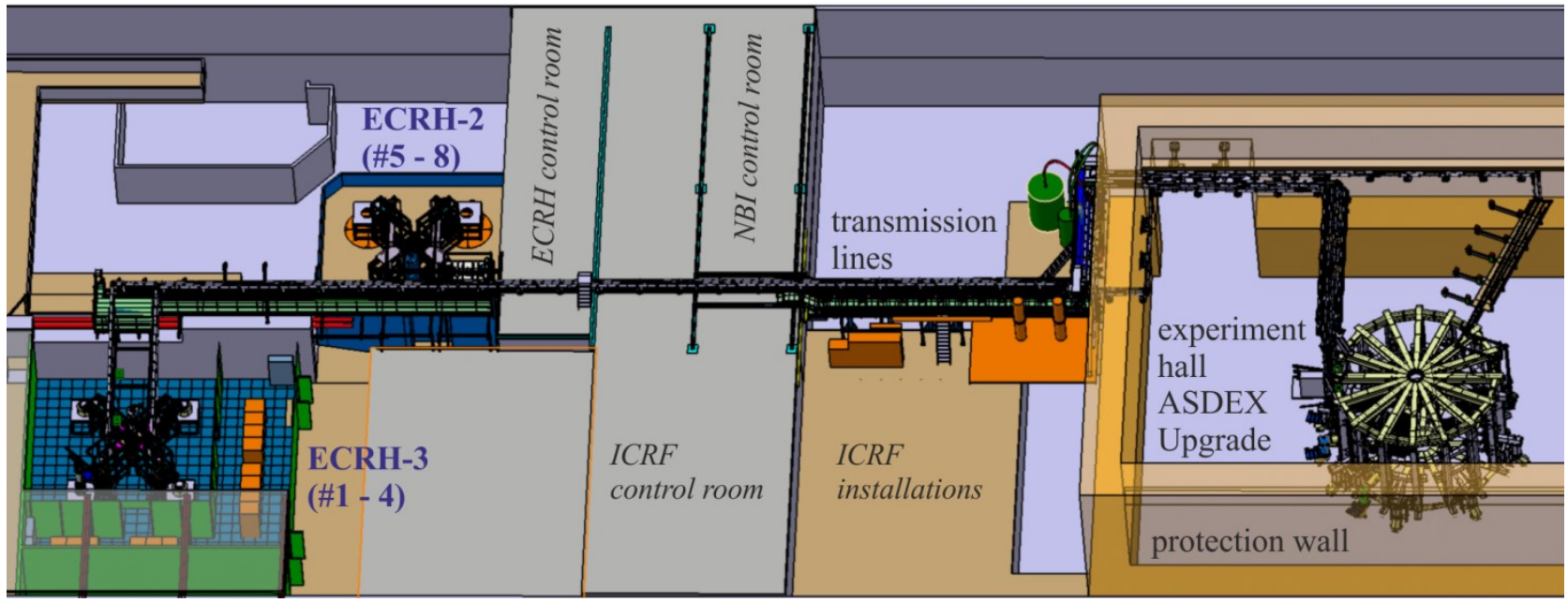

Fig. 2. Electron cyclotron resonance heating installations comprising three storeys in main ASDEX Upgrade building. Ground floor: blue, experiment hall. First floor: light brown, access to each group of four Gyrotrons ('ECRH-2,',ECRH-3'). Second floor: grey, transmission lines.

\section{Extension of ECRH installations}

An overview of ECRH launchers at AUG is given in figure 1 and the installations are shown in figure 2 . In 2016 four Gyrotrons with $10 \mathrm{~s}$ pulse length and output power close to $1 \mathrm{MW}$ per unit were available. This group of four Gyrotrons belongs to an installation which was described in [9]. Following a decision which dates back to 2011, the system is being extended to eight similar units in total. The launching positions \#1-4 (figure 1) stem from AUG's first ECRH system [10] and comments on operation experience are found in [11]. The periphery of this original ECRH system was dismantled and the launchers are now connected to the recent installations, cf. figure 2 .

In order to detect unabsorbed millimeter waves, Sniffer spheres (figure 1) were originally installed only. This was with the intention to make the measurement insensitive to polarization and phase. From the measurements described in [12] and [13] it became clear that the dynamics of the main plasma is mainly responsible for mixing the phase and polarization of the stray radiation. At AUG a simple D-band pick-up wave guide, therefore, provides basically the same signal properties [13] as the measurement via Sniffer sphere.

\section{Simulation of the 0-2 mode scheme}

An O-2 mode scheme exists for the launchers \#5 and \#6, as described in [6]. There are similar upgrades (figure 3) for \#7 and \#8. The reflector \#8 has been manufactured, tested and is ready for operation, while \#7 is intended to be prepared for the 2018 campaign. The criteria [6] for the selection of the beam geometry were basically:

(i) toroidal angle for maximum absorption [7],

(ii) poloidal/vertical angle to allow central heating,

(iii) beam should end on a robust surface after $2^{\text {nd }}$ pass.

Both angles are adapted such that the reflector fits into the tile columns of the heat shield.
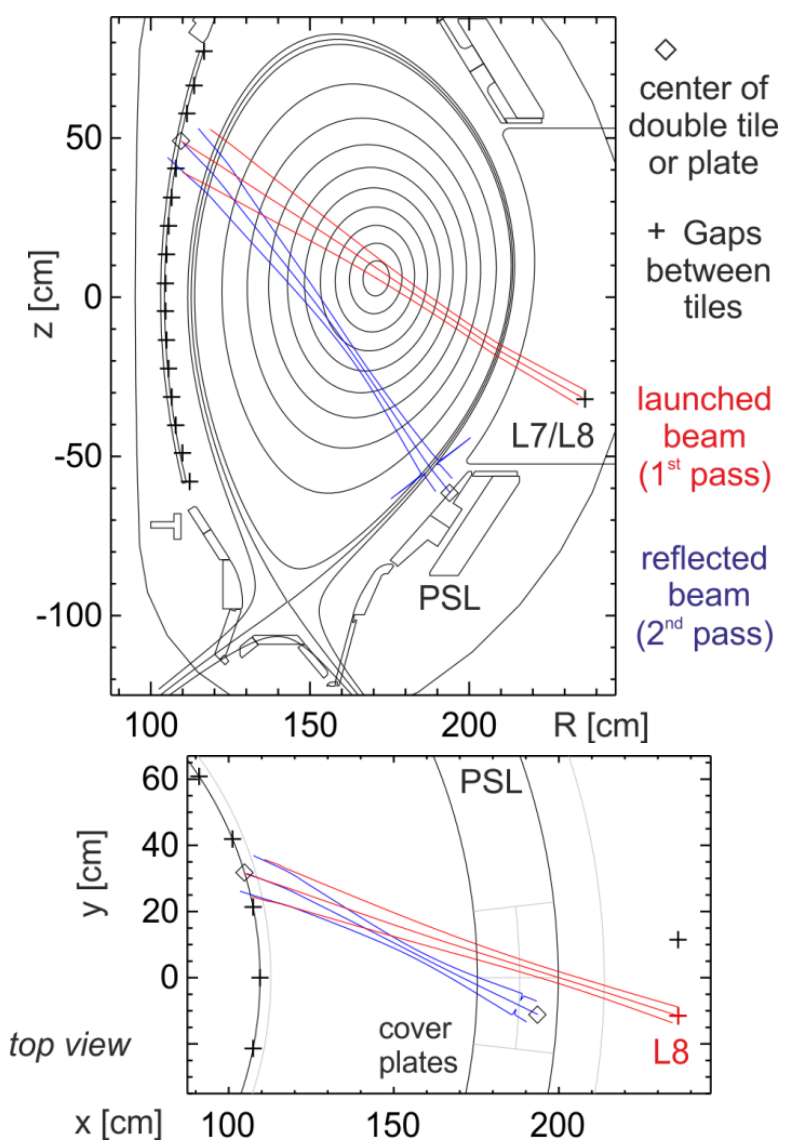

Fig. 3. Simulation of the $\mathrm{O}-2$ mode beam propagation and absorption [14]. First pass: $75 \%$ absorption, second pass: $80 \%$ giving a total of $95 \%$ of the launched power. After the $2^{\text {nd }}$ pass, spikes may occur in the simulated beam width. These are artefacts produced by insufficient smoothing in the experimental density profile, particularly in the pedestal region. Input data: AUG \#30505, $\mathrm{t}=3.3 \mathrm{~s}, I_{\mathrm{p}}=1.2 \mathrm{MA}, B_{\mathrm{t}}=-2.48 \mathrm{~T}$, central electron density $n_{\mathrm{e}}=1.15 \cdot 10^{20} \mathrm{~m}^{-3}$ and $T_{\mathrm{e}}=3.6 \mathrm{keV}$.

The robust beam facing component after the $2^{\text {nd }}$ pass is important, since the remaining millimeter wave power may contain up to $15 \%$ of the launched power. In this case the cover plates of the lower passive stabilization loop (PSL, figure 3) are considered large and robust enough. The remaining power is reflected on this plates and spreads out due to beam divergence.

The simulations shown in figure 3 are based on the Torbeam [14] beam tracing code, using data from AUG 
experiment $\# 30505$ at $\mathrm{t}=3.3 \mathrm{sec}$ with a total heating power of approx. 19.5 MW. This is considered a model high power discharge with partial detachment.

All beamlines including \#1-4 are to be equipped with appropriate reflectors on the central column by 2019 . Individual constraints arise from the launching position, the position of the appropriate beam dump, and from material and instrumentation of the holographic mirrors.

\section{Reflector design}

The plasma facing surface of the central column inside AUG is composed of tiles (figure 4) and the surface contours of each tile are designed such, that leading edges are avoided. According to the simulation result, the reflector is large enough, if a double tile of approx. $210 \times 180 \mathrm{~mm}$ is used.

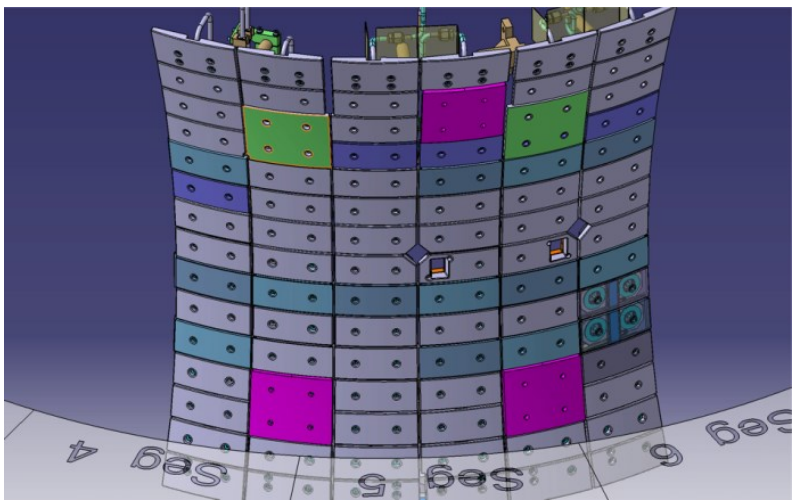

Fig. 4. Heat shield tiles in AUG vessel segments 4-6. The lower magenta double tiles are the reflectors \#5 and \#6 [6]. The upper green double tiles are the upgrades for $\# 7$ and $\# 8$ and correspond to figure 3 . The upper magenta tile in the middle is a reflector that was mounted for measurement purposes [8].

\subsection{Optimized gratings}

The reflectors need optimized gratings in order to direct the beam in the direction of the second pass through the plasma. The requirements for the grating are

(i) the direction of the reflected beam,

(ii) possibly a refocusing characteristic,

(iii) polarization independence.

Furthermore, the tiles on the high field side have typically a saddle-like surface, into which the grooves are milled. Additional constraints are imposed by the available milling tools, which limit the difference of the minimum and maximum slope angles of a grating.

The optimization is done by calculating the local $\mathbf{k}$ vectors on the reflector and approximating the fields by plane waves, which results in a $2 \mathrm{D}$ field problem. The 2D groove profiles are then optimized in order to maximize the reflected intensities $I_{n}^{\mathrm{TM}}$ and $I_{n}^{\mathrm{TE}}$ for both polarizations in the desired diffraction order $n$ and minimize the phase difference $\phi^{\mathrm{TM}-\mathrm{TE}}$ between the TM and TE polarizations. The overall efficiency becomes:

$$
\eta_{\circ}=\frac{1}{4} I_{n}^{\mathrm{TM}}+\frac{1}{4} I_{n}^{\mathrm{TE}}+\frac{1}{2} \sqrt{I_{n}^{\mathrm{TE}} I_{n}^{\mathrm{TM}}} \cos \left(\phi^{\mathrm{TM}-\mathrm{TE}}\right)
$$

The remaining power will be radiated in other diffraction orders and contributes to the stray radiation level in the vessel. The 2D gratings are optimized separately, efficiencies are calculated using a BEM algorithm [8]. The complete 3D-grating is interpolated from the $2 \mathrm{D}$ profiles [6]. The separate optimization of the $2 \mathrm{D}$ profiles can be simply parallelized, because no data needs to be transferred between the optimization processes.

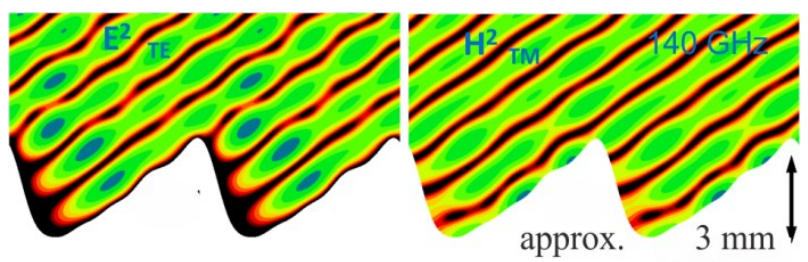

Fig. 5. Exemplary mirror grating and contours of the millimetre wave amplitude squared [8]. This $3^{\text {rd }}$ order grating with $\theta_{\text {in }}=60^{\circ}$ and $\theta_{\text {out }}=50^{\circ}$ has an efficiency of 0.89 for TE and 0.97 for TM.

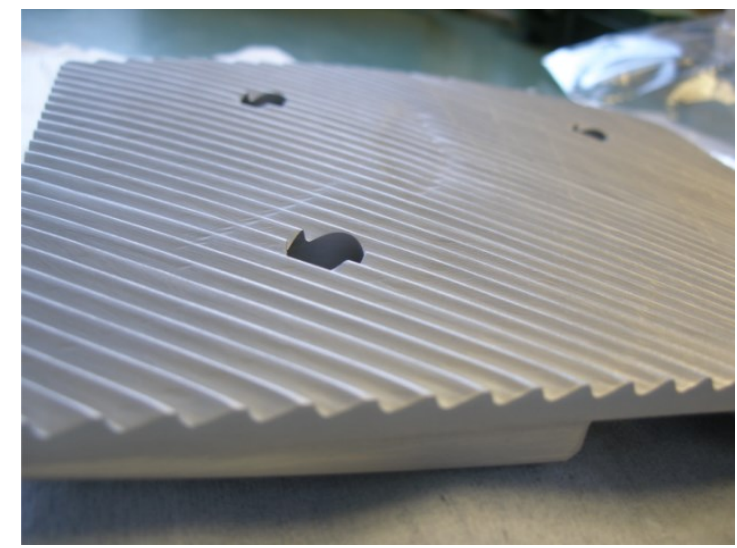

Fig. 6. Reflector $\# 8$, machined from graphite with optimized grating and coated with tungsten.

\subsection{Thermocouple instrumentation}

If the millimeter wave beam has an overlap with a gap between the tiles, part of it may penetrate into the gap and cause damage. The beam alignment on the reflector is, therefore, critical. Thermocouples built into the surface of the reflectors can be used to verify the beam position [6]. The fastest response times are achieved, if the thermocouples are flush mounted (figures 7 and 8).

During assembly (figure 9) special attention has been paid with respect to maintainability. The $\mathrm{NiCr}-\mathrm{Ni}$ element is fixated in a single spot on the rear side and it is intended to be exchangeable.

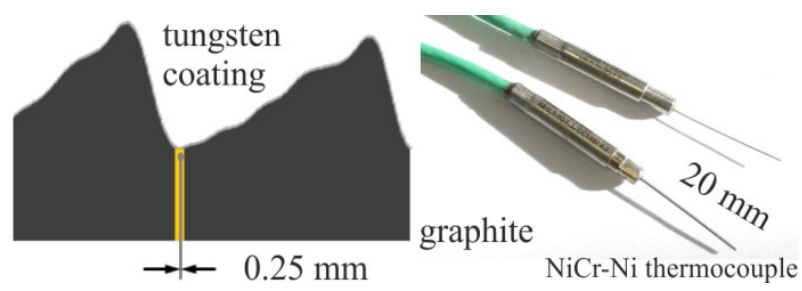

Fig. 7. Scheme of a thermocouple mount (left) in the solid body below the grating. The tip of the NiCr-Ni thermocouple (right) has to be adjusted flush with the plasma facing surface. 


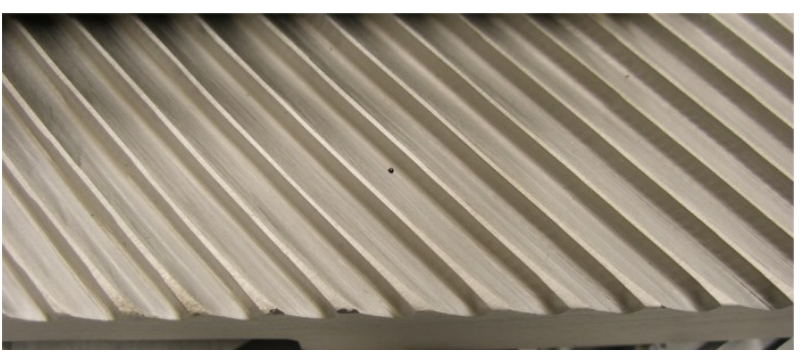

Fig. 8. Flush mounted NiCr-Ni thermocouple, its tip can be recognized as a small bright spot within the borehole. The borehole diameter of $0.5 \mathrm{~mm}$ is already in cutoff for the millimetre wave.
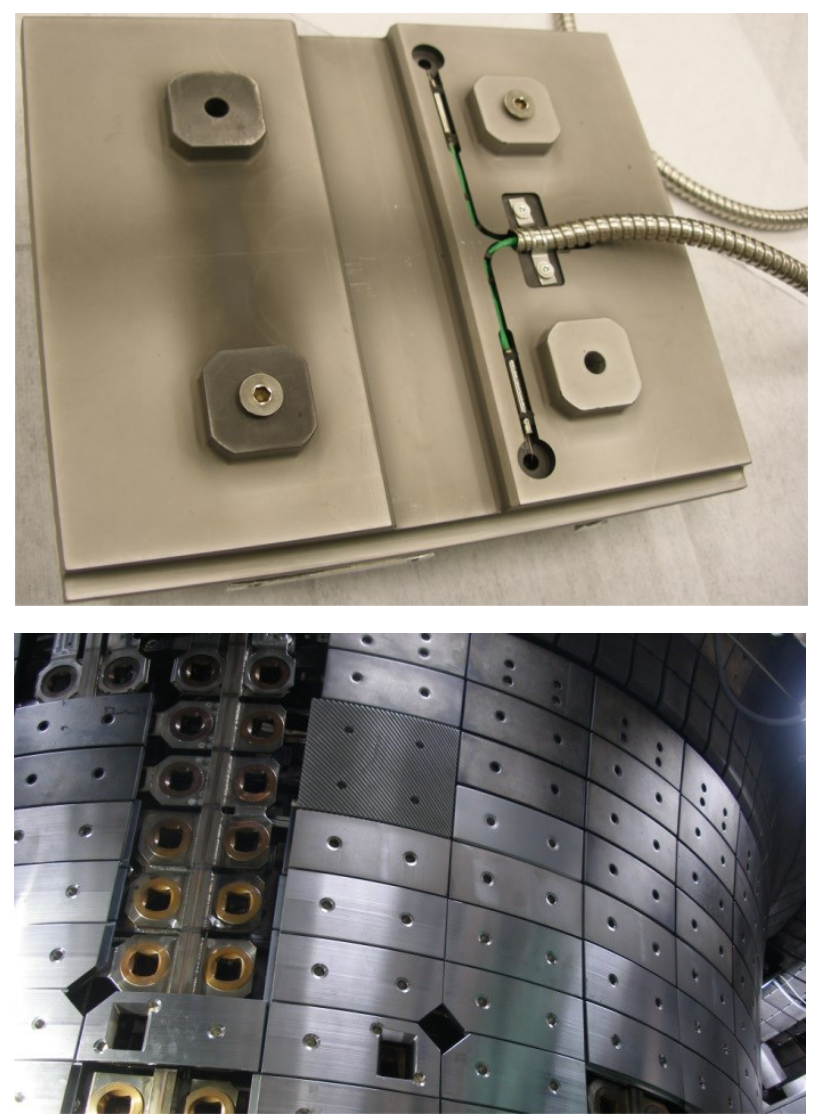

Fig. 9. Top: Grooves machined into the rear part increase the shielding of the thermocouples and their cables. In addition, cables are protected by flexible stainless steel tube. Bottom: Reflector \#8 assembly completed inside AUG.

\section{Machine protection and operation}

Considerable effort has been spent on AUG in order to harden the components on the inner column against the millimetre waves from the ECRH. An overlapping design of the tile edges (figure 10) increases the shielding against $\mathrm{mm}$-waves and reduces the risk of severe damage, if an error occurs. The shielding factor has been measured on a laboratory test mock-up and its worstcase value 0.036 or $-14.4 \mathrm{~dB}(140 \mathrm{GHz})$ is considered sufficient for this purpose. Vital parts behind the tiles have received additional tight covers.

Launcher alignment can be accurately checked in vacuum, sweeping the beam over the thermocouple positions while the Gyrotron generates a pulse train with reduced microwave power [15].

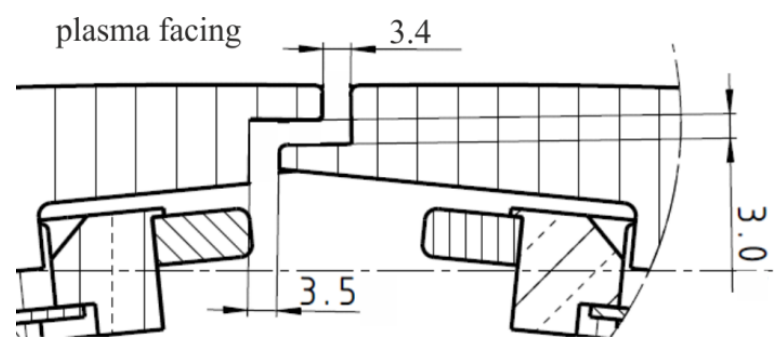

Fig. 10. The edges of the heat shield tiles on the inner column of AUG have been redesigned with a labyrinth-like dogleg structure in order to increase the shielding against millimetre waves.

During plasma operation it is found that the toroidal position of the beam on the reflector is very robust with respect to changes in the plasma configuration. The vertical position of the beam on the reflector may change in particular with plasma density [6] and an automated feedback of the launch angle, depending on the thermocouple signals was tested successfully. In the harsh environment of high power plasma operation and under certain conditions, the thermocouple signals are, however, subject to cross-talk from the AUG ICRF. It is to be tested, whether the improved shielding of the thermocouples and their cables (section 4.2) is sufficient.

Video observation is helpful in order to detect arcs, which are ignited if the beam crosses the tile edge. Automated real-time video analysis is being developed and will be discussed elsewhere.

The skillful and precise assembly by H. Schuetz is gratefully acknowledged.

This work has been carried out within the framework of the EUROfusion Consortium and has received funding from the Euratom research and training programme 2014-2018 under grant agreement No 633053. The views and opinions expressed herein do not necessarily reflect those of the European Commission.

\section{References}

1. A. Kallenbach et al, Nucl. Fusion 55, 053026 (2015)

2. A. Herrmann et al, Nucl. Fusion 55, 063015 (2015)

3. A. Kallenbach et al, Nucl. Fusion 51, 094012 (2011)

4. O. Gruber et al, Nucl. Fusion 49, 115014 (2009)

5. D. Wagner et al, J Inf Milli Terahz 37, 45-54 (2016)

6. H. Höhnle et al, Nucl. Fusion 51, 083013 (2011)

7. V. Erckmann, U. Gasparino, Plasma Phys. Control. Fusion 36, 1869-1962 (1994)

8. O. Mangold, $\mathrm{PhD}$ thesis (Stuttgart University, 2009)

9. F. Leuterer et al, Fus. Eng. Des. 66-68, 537 (2003)

10. B. Streibl et al, Fusion Sci. Technol. 44, 578 (2003)

11. F. Leuterer et al, Fusion Sci. Technol. 55, 31 (2009)

12. M. Schubert et al, EPJ Web Conf 32, 02013 (2012)

13. M. Schubert et al, EPJ Web Conf 87, 02010 (2015)

14. E. Poli, A. Peeters, G. Pereverzev, Comput. Phys. Commun. 136, 90 (2001)

15. M. Schubert et al, $43^{\text {rd }}$ Conference on Plasma Physics, ECA 40A, P1.026 (2016) 